\title{
Sharp Stability Results for Almost Conformal Maps in Even Dimensions
}

\author{
By Stefan Müller, Vladimir Šverák, and Baisheng Yan
}

\begin{abstract}
Let $\Omega \subset \mathbf{R}^{n}$ and $n \geq 4$ be even. We show that if a sequence $\left\{u^{j}\right\}$ in $W^{1, n / 2}\left(\Omega ; \mathbf{R}^{n}\right)$ is almost conformal in the sense that dist $\left(\nabla u^{j}, \mathbf{R}^{+} S O(n)\right)$ converges strongly to $O$ in $L^{n / 2}$ and if $u^{j}$ converges weakly to $u$ in $W^{1, n / 2}$, then $u$ is conformal and $\nabla u^{j} \rightarrow \nabla u$ strongly in $L_{\text {loc }}^{q}$ for all $1 \leq q<n / 2$. It is known that this conclusion fails if $n / 2$ is replaced by any smaller exponent $p$. We also prove the existence of a quasiconvex function $f(A)$ that satisfies $0 \leq f(A) \leq C\left(1+|A|^{n / 2}\right)$ and vanishes exactly on $\mathbf{R}^{+} S O(n)$. The proof of these results involves the Iwaniec-Martin characterization of conformal maps, the weak continuity and biting convergence of Jacobians, and the weak- $L^{1}$ estimates for Hodge decompositions.
\end{abstract}

\section{Introduction}

Let $n \geq 2$ and $\Omega$ be a domain in $\mathbf{R}^{n}$. We denote by $W^{1, p}\left(\Omega ; \mathbf{R}^{n}\right)(p \geq 1)$ the usual space of all Sobolev maps $u: \Omega \rightarrow \mathbf{R}^{n}$. A map $u \in W^{1, p}\left(\Omega ; \mathbf{R}^{n}\right)$ is called conformal if

$$
\nabla u(x) \in \mathbf{R}^{+} S O(n)=\{\lambda Q \mid \lambda \geq 0, Q \in S O(n)\} \text { a.e. } x \in \Omega .
$$

Here, $\mathbf{R}^{+}$denotes all nonnegative real numbers, and $S O(n)$ denotes the set of all rotations with determinant equal to 1. A classical Liouville's theorem asserts that if $n \geq 3$ and $p \geq n$, then a conformal map in $W^{1, p}\left(\Omega ; \mathbf{R}^{n}\right)$ must be a restriction onto $\Omega$ of a Möbius map (see [4] and [26]). A recent result of Iwaniec and Martin [16] shows that in even dimensions Liouville's theorem is still true for conformal maps in $W^{1, p}$ if $p \geq n / 2$. In odd dimensions, Liouville's theorem holds for conformal maps in $W^{1, p}$ if $p$ is not too far below $n$; the minimal value of all such $p^{\prime} s$ is unknown (see [14] and [17]). Note that there are counterexamples in all dimensions showing that a conformal map in $W^{1, p}$ for $p<n / 2$ may not be a restriction of a Möbius map (see, e.g., [16]).

In this paper, we are mainly interested in the stability of conformal maps, i.e., the question whether the weak limit of almost conformal maps is conformal. In the following, weak convergence is denoted by the half-arrow " $\rightarrow$ " and strong convergence by the arrow " $\rightarrow$." Our main result is the following:

Theorem 1.1. Suppose $n \geq 4$ is even and that $\left\{u^{j}\right\}$ is a sequence in $W^{1, n / 2}\left(\Omega ; \mathbf{R}^{n}\right)$ and satisfies

$$
u^{j} \rightarrow u \text { in } W^{1, n / 2}\left(\Omega ; \mathbf{R}^{n}\right)
$$

Math Subject Classifications. 30C65, 35A15.

Key Words and Phrases. quasi-conformal mappings, stability, quasi-convex functions. 
and

$$
\text { dist }\left(\nabla u^{j}, \mathbf{R}^{+} S O(n)\right) \rightarrow 0 \text { in } L^{n / 2}(\Omega) .
$$

Then $u$ is conformal (thus a Möbius map) and $\nabla u^{j} \rightarrow \nabla u$ in $L_{\text {loc }}^{q}(\Omega)$ for all $1 \leq q<n / 2$. In fact, $\nabla u^{j} \rightarrow \nabla u$ in the Marcinkiewicz space weak $-L_{\mathrm{loc}}^{n / 2}=L_{\mathrm{loc}}^{n / 2, \infty}$.

Remarks. 1. If (1.1) is replaced by

$$
u^{j} \rightarrow u \quad \text { in } W^{1, p}\left(\Omega ; \mathbf{R}^{n}\right) \text { for all } 1 \leq p<n / 2,
$$

the conclusion fails (see Example 4.2 below). Also, if $n / 2$ in both (1.1) and (1.2) is replaced by $p>n / 2$, then $\nabla u^{j} \rightarrow \nabla u$ in $L_{\text {loc }}^{p}(\Omega)$; see the remark after the proof of Theorem 1.1.

2. Hypothesis (1.2) can be replaced by the following seemingly more general condition. Let $f: \mathbf{M}^{n \times n} \rightarrow \mathbf{R}$, where $\mathbf{M}^{n \times n}$ denotes the set of all real $n \times n$ matrices, be a nonnegative $n / 2$ homogeneous continuous function that vanishes exactly on $\mathbf{R}^{+} S O(n)$ and we assume that

$$
f\left(\nabla u^{j}\right) \rightarrow 0 \text { in } L^{1}(\Omega) .
$$

This hypothesis is equivalent to the special case (1.2) under the condition (1.1) since, by homogeneity,

$$
0 \leq f(A) \leq \epsilon|A|^{n / 2}+C_{\epsilon} \operatorname{dist}^{n / 2}\left(A, \mathbf{R}^{+} S O(n)\right), \quad \forall \epsilon>0, \forall A \in \mathbf{M}^{n \times n} .
$$

A particular choice $f(A)=\|A\|^{n / 2}(1-r(A))$, where $\|A\|$ is the operator norm and $r(A)=$ $\operatorname{det} A /\|A\|^{n}$, shows that in even dimensions the weak limit of weakly $K_{j}$-quasiregular maps $u^{j}$ in $W^{1, n / 2}\left(\Omega ; \mathbf{R}^{n}\right)$ with $K_{j} \rightarrow 1$ is conformal and the convergence is in fact strong in $W_{\text {loc }}^{1, q}$ for all $1 \leq q<n / 2$ (see [14], [16], and [26]).

3. If $n$ is odd, the similar conclusion of the theorem still holds if in both (1.1) and (1.2) one replaces $n / 2$ by a number $p$ which is not too far below $n$ (see [34], [37]).

One key ingredient of the proof of the main theorem is the fact that in even dimensions conformality of a matrix can be (almost) characterized by a condition that involves only minors of order $n / 2$ (see Lemma 2.1). This characterization, which may be viewed as a nonlinear version of the Cauchy-Riemann equations, is due to Donaldson and Sullivan [10] for $n=4$ and to Iwaniec and Martin [16] for the general case $n=2 l, l \geq 2$.

The stability result stated in Theorem 1.1 is closely related to the existence of a quasiconvex function $f$ that vanishes exactly on the set $\mathbf{R}^{+} S O(n)$ and satisfies the growth condition $0 \leq f(A) \leq$ $C\left(1+|A|^{n / 2}\right)$. If $n / 2$ is replaced by $p \geq n$, then such functions exist, as is easily seen [3], [19] by considering the function $f(A)=|A|^{n}-n^{n / 2} \operatorname{det} A$, where $|A|$ is the norm defined by $|A|^{2}=\operatorname{tr}\left(A^{t} A\right)$.

Recall that a function $f: \mathbf{M}^{n \times n} \rightarrow \mathbf{R}$ is said to be quasiconvex if for a bounded smooth domain $D \subset \mathbf{R}^{n}$

$$
\int_{D} f(A) d x \leq \int_{D} f(A+\nabla \phi(x)) d x, \quad \forall A \in \mathbf{M}^{n \times m}, \forall \phi \in C_{0}^{\infty}\left(D ; \mathbf{R}^{n}\right) .
$$

Furthermore, the quasiconvexification of $f$, denoted by $f^{q c}$, is defined by

$$
f^{q c}(A)=\inf _{\phi \in C_{0}^{\infty}\left(D, \mathbf{R}^{n}\right)} \frac{1}{|D|} \int_{D} f(A+\nabla \phi(x)) d x .
$$


A simple covering argument shows that the definition of both quasiconvexity and quasiconvexification is independent of the domain $D$. Moreover, under suitable growth conditions, $f^{q c}$ is the largest quasiconvex function on $\mathbf{M}^{n \times n}$ below $f$. However, most quasiconvex functions that are explicitly known are polyconvex functions introduced by Ball [2], i.e., functions that can be expressed as a convex function of all minors (subdeterminants) of $A$. For further information about quasiconvexity, we refer to [1], [6], [8], [20], [22], and [28].

Theorem 1.2. Let $n \geq 2$ be even. Then there exists a quasiconvex function $f$ which satisfies

$$
\left\{\begin{array}{c}
0 \leq f(A) \leq C\left(1+|A|^{n / 2}\right), \forall A \in \mathbf{M}^{n \times n} \\
f(A)=0 \text { if and only if } A \in \mathbf{R}^{+} S O(n)
\end{array}\right.
$$

Furthermore, the function $f$ may be taken to be $n / 2$-homogeneous.

To put this result in perspective, we first remark that the result for $n=2$ follows from the standard compensated compactness argument using the div-curl lemma (see [24], [30], [33], [36]). Second, as proved in [35], there are no polyconvex functions that satisfy condition (1.5), and also the growth condition $0 \leq f(A) \leq C\left(1+|A|^{n / 2}\right)$ in (1.5) cannot be strengthened to $0 \leq f(A) \leq$

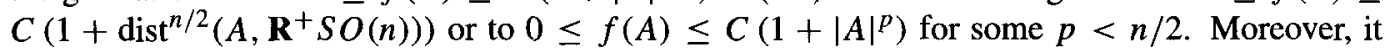
follows from a result of [39] that whether there exists a quasiconvex function which vanishes exactly on a compact subset $\mathcal{K}$ of $\mathbf{M}^{n \times n}$ does not depend on the growth condition of the quasiconvex function (see also [36]). Finally, whether or not Theorem 1.2 holds in odd dimensions is open.

The construction of suitable quasiconvex functions plays an important rôle in the calculus of variations and nonlinear partial differential equations; see [9], [11], and [32]. Applications to phase transformations in elastic crystals have recently attracted considerable attention and we refer to Ball and James [5], Chipot and Kinderlehrer [7], Fonseca [12], Kohn [21], Müller and Šverák [25], and Šverák [29], [31] for further information.

\section{Notation and preliminaries}

We first recall some well-known results concerning multilinear algebra and differential forms. We follow the notation of [14] and [16].

Let $e_{1}, e_{2}, \cdots, e_{n}$ denote the standard basis of $\mathbf{R}^{n}$. For $k=0,1, \cdots, n$ we denote by $\Lambda^{k}=$ $\wedge^{k}\left(\mathbf{R}^{n}\right)$ the linear space of all $k$-tensors spanned by $\left\{e_{I}=e_{i_{1}} \wedge e_{i_{2}} \wedge \cdots \wedge e_{i_{k}}\right\}$ for all ordered $k$-tuples $I=\left(i_{1}, i_{2}, \cdots, i_{k}\right)$ with $1 \leq i_{1}<i_{2}<\cdots<i_{k} \leq n$. Define $\Lambda^{k}=\{0\}$ if $k<0$ or $k>n$. The Grassmann algebra $\Lambda=\oplus \Lambda^{k}$ is a graded algebra with respect to the exterior multiplication $\wedge$.

For $\alpha=\sum{ }_{I} \alpha_{I} e_{I}$ and $\beta_{I}=\sum \beta_{I} e_{I}$ in $\Lambda$ the inner product is defined by

$$
\langle\alpha, \beta\rangle=\sum_{I} \alpha_{I} \beta_{I}
$$

where summation is taken over all $k$-tuples $I=\left(i_{1}, i_{2}, \cdots, i_{k}\right)$ and all integers $k=0,1, \cdots, n$. The norm of $\alpha \in \Lambda$ is defined by $|\alpha|^{2}=\langle\alpha, \alpha\rangle$. The Hodge star operator $*: \Lambda \rightarrow \Lambda$ is defined by requiring

$$
* 1=e_{1} \wedge e_{2} \wedge \cdots \wedge e_{n}
$$

and

$$
\alpha \wedge(* \beta)=\beta \wedge(* \alpha)=\langle\alpha, \beta\rangle(* 1)
$$

for all $\alpha, \beta \in \Lambda$. It is obvious that $*$ maps $\Lambda^{k}$ into $\Lambda^{n-k}$. 
For a linear operator $A: \mathbf{R}^{n} \rightarrow \mathbf{R}^{n}$, the $k$ th exterior power $\wedge^{k} A=A_{\#}^{k}$ of $A$ is defined as a map $A_{\#}^{k}: \Lambda^{k} \rightarrow \Lambda^{k}$ by

$$
A_{\#}^{k}\left(x_{1} \wedge x_{2} \wedge \cdots \wedge x_{k}\right)=A x_{1} \wedge A x_{2} \wedge \cdots \wedge A x_{k}, \quad x_{j} \in \mathbf{R}^{n} .
$$

Note that if $A$ has the $n \times n$ matrix form in the standard basis $\left\{e_{1}, e_{2}, \cdots, e_{n}\right\}$, then $A_{\#}^{k}$ has the $\left(\begin{array}{l}n \\ k\end{array}\right) \times\left(\begin{array}{l}n \\ k\end{array}\right)$ matrix form in the basis $\left\{e_{I}=e_{i_{1}} \wedge e_{i_{2}} \wedge \cdots \wedge e_{i_{k}}\right\}$ for all ordered $k$-tuples $I=\left(i_{1}, i_{2}, \cdots, i_{k}\right)$ with $1 \leq i_{1}<i_{2}<\cdots<i_{k} \leq n$ and each element of $A_{\sharp}^{k}$ is a $k \times k$ minor of $A$.

For the purpose of this paper, we shall assume $n=2 l$ and consider only the $l$ th exterior power $A_{\#}^{l}$ of an $n \times n$ matrix $A$. In this case, the Hodge $*$ operator induces a linear operator from $\Lambda^{l}$ onto itself. Define $W: \mathbf{M}^{n \times n} \rightarrow \mathbf{R}$ as follows:

$$
W(A)=\left\|*\left(A^{t}\right)_{\text {\# }}^{l}-\left(A^{t}\right)_{\text {米 }}^{l} *\right\|,
$$

where the norm $\|\cdot\|$ is taken to be the operator norm. Note that $W(A)$ is polyconvex and hence quasiconvex.

The following result is the Iwaniec-Martin characterization of conformal matrices.

Lemma 2.1 [16]. Let $W(A)$ be defined as above and $Z(W)$ denote the zero set of $W$. Then $W(A)$ is polyconvex and

$$
Z(W)=K_{n} \cup\left\{A \in \mathbf{M}^{n \times n} \mid \operatorname{rank} A \leq l-1\right\} .
$$

Proof. This follows from Lemmas 2.10 and 2.13 of Iwaniec and Martin [16].

Lemma 2.2. Let $I=\left(i_{1}, i_{2}, \cdots, i_{l}\right)$ with $1 \leq i_{1}<i_{2}<\cdots<i_{l} \leq n$ be given. For any $A \in \mathbf{M}^{n \times n}$ with row vectors $a_{j}$ being considered as in $\Lambda^{1}$. Define a matrix $P_{I}(A) \in \mathbf{M}^{l \times n}$ such that its $k$ th row is $a_{i_{k}}$ for $k=1, \ldots, l$. Then it follows that

$$
\left|P_{l}(A)\right|^{l} \geq l^{l / 2}\left|\left(A^{t}\right)_{\#}^{l}\left(e_{I}\right)\right|,
$$

and equality holds in (2.2) if and only if there exists a $\lambda_{I} \geq 0$ such that

$$
a_{i_{k}} \cdot a_{i_{s}}=\lambda_{I} \delta_{k s}
$$

for all $1 \leq k, s \leq l$. Therefore, equality in (2.2) holds for all indices $I$ if and only if $A$ is a conformal or an anticonformal matrix, i.e., $A^{t} A=\lambda I_{n}$ for some $\lambda \geq 0$.

Proof. This is a special case of the so-called Hadamards' inequality in the theory of matrices. See, for example, [15, Lemma 2.1].

We now review the notation of differential forms on $\Omega$. For each $k=0,1, \cdots, n$, a differential form $\alpha$ of degree $k$ defined on $\Omega$ (denoted by $\alpha \in \Lambda^{k}(\Omega)$ )

$$
\alpha=\sum \alpha_{I}(x) d x_{I}=\sum \alpha_{i_{1} i_{2} \ldots i_{k}}(x) d x_{i_{1}} \wedge d x_{i_{2}} \wedge \cdots \wedge d x_{i_{k}}
$$

can be identified with a function $\alpha: \Omega \rightarrow \Lambda^{k}\left(\mathbf{R}^{n}\right)$ with the same coefficients $\left\{\alpha_{I}\right\}$. Consider the space

$$
\mathcal{D}^{\prime}(\Omega ; \Lambda)=\oplus_{k} \mathcal{D}^{\prime}\left(\Omega ; \Lambda^{k}\right)
$$


of all differential forms whose coefficients are distributions on $\Omega$. Similarly, other spaces such as $L^{p}(\Omega ; \Lambda), W^{1, p}(\Omega ; \Lambda)$ can be defined by requiring that all coefficients belong to the suitable function spaces. by

Recall that the exterior derivative $d: \mathcal{D}^{\prime}\left(\Omega ; \Lambda^{k}\right) \rightarrow \mathcal{D}^{\prime}\left(\Omega ; \Lambda^{k+1}\right)$ for $k=0,1, \ldots, n$ is defined

$$
\begin{aligned}
d \alpha & =\sum_{I} d \alpha_{I}(x) \wedge d x_{I} \\
& =\sum_{s=1}^{n} \sum_{1 \leq i_{1}<i_{2}<\cdots<i_{l} \leq n} \frac{\partial \alpha_{i_{1} i_{2} \ldots i_{k}}(x)}{\partial x_{s}} d x_{s} \wedge d x_{i_{1}} \wedge d x_{i_{2}} \wedge \cdots \wedge d x_{i_{k}},
\end{aligned}
$$

and its formal adjoint operator $d^{*}: \mathcal{D}^{\prime}\left(\Omega ; \Lambda^{k+1}\right) \rightarrow \mathcal{D}^{\prime}\left(\Omega ; \Lambda^{k}\right)$ (the so-called Hodge codifferential) is given by $d^{*}=(-1)^{n k+1} * d *$ on $(k+1)$-forms. It follows directly from definition that the Laplace-Beltrami operator $\Delta=d d^{*}+d^{*} d$ defined on $\mathcal{D}^{\prime}\left(\Omega ; \Lambda^{k}\right)$ operates only on the coefficients, i.e.,

$$
\Delta \alpha(x)=\sum_{I} \Delta \alpha_{I}(x) d x_{I}, \quad \text { where } \Delta=-\sum_{i} \frac{\partial^{2}}{\partial x_{i}^{2}} .
$$

Let $u: \Omega \rightarrow \mathbf{R}^{n}, u=\left(u_{1}, u_{2}, \cdots, u_{n}\right)$ be a map in $W_{\text {loc }}^{1, k p}$. Then $u$ induces a homomorphism

$$
u^{*}: C^{\infty}\left(\mathbf{R}^{m}, \Lambda^{k}\right) \rightarrow L_{\mathrm{loc}}^{p}\left(\Omega ; \Lambda^{k}\right)
$$

which is defined as follows. For each $\alpha=\sum_{I} \alpha_{I}(x) d x_{I} \in C^{\infty}\left(\mathbf{R}^{m}, \Lambda^{k}\right)$, let

$$
\left(u^{*} \alpha\right)(x)=\sum_{I} \alpha_{I}(u(x)) d u_{i_{1}} \wedge d u_{i_{2}} \wedge \cdots \wedge d u_{i_{k}} .
$$

If $\alpha$ has constant coefficients, then $\left(u^{*} \alpha\right)(x)$ can be identified with the $k$ th exterior power of $\nabla^{t} u(x)$, where $\nabla^{t} u(x)$ denotes the transpose of $\nabla u(x) \in \mathbf{M}^{n \times n}$. Thus, in our notation

$$
\left(u^{*} \alpha\right)(x)=\left(\nabla^{t} u(x)\right)_{\#}^{k} \alpha .
$$

We use the following weak- $L^{1}$ estimates for the Hodge decomposition.

Lemma 2.3. Let $D$ be any smooth domain in $\mathbf{R}^{n}$. Suppose $\omega \in L^{1}\left(D ; \Lambda^{k}\right)$ and $\alpha \in L_{\text {loc }}^{1}\left(D ; \Lambda^{k}\right)$. If the system

$$
d \alpha=0, \quad d^{*} \alpha=d^{*} \omega
$$

holds in the sense of distributions on $D$, then there exists a harmonic form $h$ on $D$ such that the weak- $L^{1}$ estimate

$$
\|\alpha-h\|_{L_{w}^{1}(D)} \leq C(n)\|\omega\|_{L^{1}(D)}
$$

holds, where $\|\gamma\|_{L_{w}^{1}(D)}$ or in general $\|\gamma\|_{L_{w}^{p}(D)}$ denotes the pseudo-norm of a form $\gamma$ in the Marcinkiewicz space weak- $L^{p}(D)=L_{w}^{p}(D)$ defined by:

$$
\|\gamma\|_{L_{w}^{p}(D)}^{p}=\sup _{t>0} t^{p} \text { meas }\{x \in D|| \gamma(x) \mid>t\}
$$

Proof. Let $\psi=N(\omega)$ be the Newton potential of $\omega$ on $D$ (defined for each coefficient of $\omega$ )

$$
\psi(x)=c_{n} \int_{D} \frac{\omega(y)}{|x-y|^{n-2}} d y
$$


and define

$$
\mathcal{R}_{i j}(\omega)=\frac{\partial^{2} \psi}{\partial x_{i} \partial x_{j}} \quad(i, j=1,2, \cdots, n) .
$$

The operator $\mathcal{R}_{i j}: \omega \rightarrow \mathcal{R}_{i j}(\omega)$ is a singular integral operator of Calderon-Zygmund type (see [13] and [27]).

From (2.4) and the identity

$$
\omega=\Delta \psi=d d^{*} \psi+d^{*} d \psi
$$

it follows that $h=\alpha-d d^{*} \psi$ is a harmonic form on $D$ and hence (2.5) follows directly from the weak- $L^{1}$ estimates for Newtonian potentials (see, e.g., [13, Ch. 9]).

Remarks. 1. If $\omega$ belongs to $L^{p}\left(D ; \Lambda^{k}\right)$ for some $1<p<\infty$, then (2.5) can be replaced by the strong $L^{p}$ estimate

$$
\|\alpha-h\|_{L^{p}(D)} \leq C(n)\|\omega\|_{L^{p}(D)} .
$$

2. If there is a constant form $\bar{\alpha}$ such that both $\omega$ and $\alpha-\bar{\alpha}$ are compactly supported in $D$, then $h \equiv \bar{\alpha}$ in both (2.5) and (2.8).

\section{3. $W^{1, p}$-stability of almost conformal maps}

In this section, we prove our main result, Theorem 1.1, about the sharp stability for almost conformal maps in even dimensions. We will frequently use the following fact.

Proposition 3.1. Let $f(\xi)$ and $g(\xi)$ be two nonnegative continuous functions on $\mathbf{R}^{N}$ that are $k$-homogeneous. If the zero set of $g$ contains that of $f$, then for every $\epsilon>0$ there exists a constant $C_{\epsilon}<\infty$ such that

$$
0 \leq g(\xi) \leq \epsilon|\xi|^{k}+C_{\epsilon} f(\xi), \quad \forall \xi \in \mathbf{R}^{N} .
$$

Proof. Let $c_{\epsilon}=\inf \{f(\xi)|| \xi \mid=1, g(\xi) \geq \epsilon\}$. Then the infimum is attained and $c_{\epsilon}>0$ by assumption. The choice $C_{\epsilon}=\sup _{|\xi|=1} g(\xi) / c_{\epsilon}$ and homogeneity yield (3.1).

Proof of Theorem 1.1. It suffices to establish the conclusion of Theorem 1.1 for smooth bounded subdomains of $\Omega$ so we may assume that $\Omega$ itself is smooth and bounded. To simplify notation let

$$
l=n / 2, \quad d(A)=\operatorname{dist}\left(A, \mathbf{R}^{+} S O(n)\right),
$$

and let $W(A)$ be defined by $(2.1)$. We proceed with the proof in several steps.

Step 1. (Strong convergence of $l \times l$ minors).

Since $W$ vanishes on conformal matrices Lemma 2.1, thus, Proposition 3.1, the boundedness of $\left\{\nabla u^{j}\right\}$ in $L^{n / 2}(\Omega)$, and (1.2) imply that

$$
W\left(\nabla u^{j}\right) \rightarrow 0 \quad \text { in } L^{1}(\Omega)
$$

Let $I=\left\{i_{1}, \cdots, i_{l}\right\}, \quad 1 \leq i_{1}<\cdots<i_{l} \leq n$, be an arbitrary $l$-index and define

$$
\omega^{j}=*\left(u^{j}\right)^{*}\left(d x_{I}\right)-\left(u^{j}\right)^{*}\left(* d x_{I}\right) .
$$


Since $\left|\omega^{j}(x)\right| \leq W\left(\nabla u^{j}(x)\right)$ a.e. (3.2) implies $\omega^{j} \rightarrow 0$ in $L^{1}\left(\Omega ; \Lambda^{l}\right)$ as $j \rightarrow \infty$. Now by (3.3)

$$
d^{*}\left[\left(u^{j}\right)^{*}\left(* d x_{I}\right)\right]=-d^{*} \omega^{j}, \quad d\left[\left(u^{j}\right)^{*}\left(* d x_{I}\right)\right]=0,
$$

and by the weak- $L^{1}$ estimates for the Hodge decomposition Lemma 2.3 there exists a harmonic $l$-form $\gamma^{j}$ in $\Omega$ such that

$$
\left\|\left(u^{j}\right)^{*}\left(* d x_{I}\right)-\gamma^{j}\right\|_{L_{w}^{1}(\Omega)} \leq C(n)\left\|\omega^{j}\right\|_{L^{1}(\Omega)} \rightarrow 0 .
$$

In particular, $\left\{\gamma^{j}\right\}$ is bounded in $L_{w}^{1}(\Omega)$ as $u^{j}$ is bounded in $W^{1, n / 2}(\Omega)$. Since $\gamma^{j}$ is harmonic in $\Omega$, thus weak- $L^{1}$ estimates imply that $\left\{\gamma^{j}\right\}$ is bounded in $C_{\mathrm{loc}}^{k}(\Omega)$ for all $k=1,2, \ldots$ and hence

$$
\gamma^{j} \rightarrow \gamma \quad \text { in } C_{\mathrm{loc}}^{k}(\Omega)
$$

and $\gamma$ is also harmonic in $\Omega$. From this and (3.5) we have for $U \subset \subset \Omega$

$$
\left\|\left(u^{j}\right)^{*}\left(* d x_{I}\right)-\gamma\right\|_{L_{w}^{1}(U)} \rightarrow 0 \text {. }
$$

On the other hand, by a result of Zhang [38] there exist decreasing measurable sets $E_{k}$ in $\Omega$ with $\left|E_{k}\right| \rightarrow 0$ such that for all $k=1,2, \cdots$

$$
\left(u^{j}\right)^{*}\left(* d x_{I}\right) \rightarrow u^{*}\left(* d x_{I}\right) \quad \text { in } L^{1}\left(\Omega \backslash E_{k}\right)
$$

as $j \rightarrow \infty$. This and (3.6) imply $\gamma=u^{*}\left(* d x_{I}\right)$ almost everywhere and hence

$$
\left\|\left(u^{j}\right)^{*}\left(* d x_{I}\right)-u^{*}\left(* d x_{I}\right)\right\|_{L_{w}^{1}(U)} \rightarrow 0
$$

for all $U \subset \subset \Omega$ and all $l$-indices $I$. Since the convergence in weak- $L_{\text {loc }}^{1}$ implies the strong convergence in $L_{\mathrm{loc}}^{s}$ for $s<1$, we deduce the strong convergence of all $l \times l$ minors in $L_{\text {loc }}^{s}(\Omega)$ for all $0<s<1$.

Step 2. (Strong convergence of $\left\{\nabla u^{j}\right\}$ ).

Let $I_{*}$ be the complementary index of $I$, i.e., $* d x_{I}=d x_{I_{*}}$. Let $1<q<l$, then Lemma 2.2 and Proposition 3.1, applied with $g(A)=\left|P_{I_{*}}(A)\right|^{q}-l^{q / 2}\left|\left(A^{t}\right)_{\#}^{l}\left(e_{I_{*}}\right)\right|^{q / l}$ and $f(A)=d^{q}(A)$, yield

$$
\left|P_{I_{*}} \nabla u^{j}\right|^{q} \leq l^{q / 2}\left|\left(u^{j}\right)^{*}\left(* d x_{I}\right)\right|^{q / l}+\epsilon\left|\nabla u^{j}\right|^{q}+C_{\epsilon, q} d^{q}\left(\nabla u^{j}\right) .
$$

We deduce from (1.2), (3.7), (3.8), and another application of Lemma 2.2 that

$$
\limsup _{j \rightarrow \infty} \int_{U}\left|P_{I_{*}} \nabla u^{j}\right|^{q} \leq \int_{U}\left|P_{I_{*}} \nabla u\right|^{q} .
$$

Summation over all $l$-indices $I$ yields the same estimate for $\left\|\nabla u^{j}\right\|_{L^{q}(U)}^{q}$ and $\|\nabla u\|_{L^{q}(U)}^{q}$. On the other hand, $\nabla u^{j} \rightarrow \nabla u$ in $L^{q}(U)$, hence,

$$
\nabla u^{j} \rightarrow \nabla u \text { strongly in } L^{q}(U) \text { for all } q<l \text { and all } U \subset \subset \Omega .
$$

In particular, $d\left(\nabla u^{j}\right) \rightarrow d(\nabla u)$ strongly in $L^{q}(U)$ which yields $\nabla u(x) \in \mathbf{R}^{+} S O(n)$ and $u$ is conformal, hence, Iwaniec-Martin's theorem shows that $u$ is a restriction onto $\Omega$ of a Möbius map [16]. 
Step 3. (Convergence of $\left\{\nabla u^{j}\right\}$ in $L_{w}^{n / 2}$ ).

In order to establish (locally) strong convergence in weak- $L^{l}$, we observe that for $q=l$ the above considerations yield

$$
h^{j}=\left(\left|\nabla u^{j}\right|^{l}-|\nabla u|^{l}\right)^{+} \rightarrow 0 \text { in } L_{w}^{1}(U)
$$

where $f^{+}$denotes the positive part of a function $f$. Let

$$
\lambda^{j}(t)=\operatorname{meas}\left\{x \in U|| \nabla u^{j}(x)-\nabla u(x) \mid>t\right\} .
$$

We have to estimate the weak- $L^{l}$ pseudo-norm

$$
\left\|\nabla u^{j}-\nabla u\right\|_{L_{w}^{l}(U)}=\left(\sup _{t \geq 0} t^{l} \lambda^{j}(t)\right)^{1 / l} .
$$

Note that, by (3.9), for all $s>0$

$$
\limsup _{j \rightarrow \infty} \sup _{t \leq s} t^{l} \lambda^{j}(t) \leq s^{l-q} \limsup _{j \rightarrow \infty} \sup _{t \geq 0} t^{q} \lambda^{j}(t)=0 .
$$

On the other hand,

$$
\begin{aligned}
\lambda^{j}(t) & \leq \operatorname{meas}\left(\left\{\left|\nabla u^{j}-\nabla u\right|>t\right\} \cap\{|\nabla u| \leq t / 3\}\right)+\operatorname{meas}\{|\nabla u|>t / 3\} \\
& \leq \operatorname{meas}\left\{h^{j} \geq(t / 3)^{l}\right\}+\text { meas }\{|\nabla u|>t / 3\}
\end{aligned}
$$

thus

$$
t^{l} \lambda^{j}(t) \leq C\left\|h^{j}\right\|_{L_{w}^{1}(U)}+C \int_{|\nabla u|>t / 3}|\nabla u|^{l}
$$

Combining this inequality with (3.10) and (3.12) we obtain, for every $s>0$

$$
\limsup _{j \rightarrow \infty}\left\|\nabla u^{j}-\nabla u\right\|_{L_{w}^{l}(U)}^{l}=\limsup _{j \rightarrow \infty} \sup _{t \geq s} t^{l} \lambda^{j}(t) \leq C \int_{|\nabla u|>s / 3}|\nabla u|^{l} .
$$

Letting $s \rightarrow \infty$, we obtain

$$
\underset{j \rightarrow 0}{\limsup }\left\|\nabla u^{j}-\nabla u\right\|_{L_{w}^{l}(U)}^{l}=0,
$$

hence Step 3 is proved. The proof of Theorem 1.1 is now complete.

Remark. If we assume $u^{j} \rightarrow u$ in $W^{1, p}$ and $d\left(\nabla u^{j}\right) \rightarrow 0$ in $L^{p}$ for some $p>n / 2$, then by Remark (1) following Lemma 2.3 one has $\left(u^{j}\right)^{*} d x_{I} \rightarrow u^{*} d x_{I}$ in $L^{p / l}$ and then easily deduces $L_{\text {loc }}^{p}$ convergence of $\left\{\nabla \boldsymbol{u}^{j}\right\}$.

\section{Quasiconvex functions that vanish exactly on $\mathbf{R}^{+} S O(n)$} Define

As before, let $d(A)$ be the distance function dist $\left(A, \mathbf{R}^{+} S O(n)\right)$ and $W(A)$ as defined by $(2.1)$.

$$
F(A)=W(A)+d^{n / 2}(A), \quad I(u)=\int_{\Omega} F(\nabla u(x)) d x .
$$


Theorem 4.1. Let $n \geq 4$ be even. Then $F^{q c}$ is nonnegative quasiconvex, $n / 2$-homogeneous and vanishes exactly on $\mathbf{R}^{+} S O(n)$. More precisely, for each $A \in \mathbf{M}^{n \times n}$ and each $\left\{\phi^{j}\right\} \subset C_{0}^{\infty}\left(\Omega ; \mathbf{R}^{n}\right)$, if $I\left(A x+\phi^{j}(x)\right) \rightarrow 0$ then $A \in \mathbf{R}^{+} S O(n)$ and $\left\|\nabla \phi^{j}\right\|_{L_{w}^{n / 2}(\Omega)} \rightarrow 0$. If $A=0$, then one has the estimate

$$
I(\phi) \geq c\|\nabla \phi\|_{L_{w}^{n / 2}(\Omega)}^{n / 2}
$$

for all $\phi \in C_{0}^{\infty}\left(\Omega ; \mathbf{R}^{n}\right)$, where $c>0$ is a constant independent of $\phi$.

Remarks. 1. In (4.2) the weak- $L^{n / 2}$ pseudo-norm cannot be replaced by the $L^{n / 2}$ norm (see Example 4.2 below).

2. If $G(A)=\left(|A|^{n}-n^{n / 2} \operatorname{det} A\right)^{1 / 2}$, it is easily seen that $W(A) \leq C G(A)$ for all $A \in \mathbf{M}^{n \times n}$. Hence, the similar proof given below also shows that the zero set $Z\left(G^{q c}\right)=\mathbf{R}^{+} S O(n)$ if $n$ is even, thus $G^{q c}(A)$ gives another quasiconvex function which satisfies the conditions of Theorem 1.2. However, all such quasiconvex functions cannot be polyconvex if $n \geq 4$ [35].

Proof of Theorem 4.1. Suppose that for some $A \in \mathbf{M}^{n \times n}$ and some sequence $\left\{\phi^{j}\right\} \subset C_{0}^{\infty}\left(\Omega ; \mathbf{R}^{n}\right)$ we have $I\left(A x+\phi^{j}(x)\right) \rightarrow 0$. We will show

$$
A \in \mathbf{R}^{+} S O(n), \quad\left\|\nabla \phi^{j}\right\|_{L_{w}^{n / 2}(\Omega)} \rightarrow 0
$$

and that if $A=0$ (4.2) holds. To this end, let $u^{j}(x)=A x+\phi^{j}(x)$. Then $I\left(u^{j}\right) \rightarrow 0$, hence, $W(A)=0$ as $\phi^{j} \in C_{0}^{\infty}\left(\Omega ; \mathbf{R}^{n}\right)$ and $W$ is quasiconvex. Define $\omega^{j}$ as in Step 1 of the proof of Theorem 1.1 by (3.3). Then $\omega^{j}$ is compactly supported in $\Omega$ since $W(A)=0$. Arguing as in Step 1 of the proof of Theorem 1.1 and taking into account Remark (2) after Lemma 2.3 we deduce that (with $l=n / 2$ as before)

$$
\left(u^{j}\right)^{*}\left(* d x_{I}\right) \rightarrow\left(A^{t}\right)_{\#}^{l}\left(* e_{I}\right) \quad \text { in } L_{w}^{l}(\Omega)
$$

Similarly, (3.8) in Step 2 of the proof of Theorem 1.1 also holds. Summation over all $l$-indices $I$ in (3.8) for $q=l$ and a sufficiently small choice of $\epsilon>0$ show that $\left\{\nabla u^{j}\right\}$ is bounded in $L_{w}^{l}(\Omega)$. As before one deduces from (3.8) and (4.3)

$$
\limsup _{j \rightarrow \infty} \int_{\Omega}\left|\nabla u^{j}\right|^{q} \leq \int_{\Omega}|A|^{q}, \forall q<l .
$$

Since $A=\frac{1}{|\Omega|} \int_{\Omega} \nabla u^{j}(x) d x$, the last inequality and strict convexity of the map $A \rightarrow|A|^{q}$ imply $\nabla u^{j} \rightarrow A$ strongly in $L^{q}(\Omega)$ for all $1<q<l$ hence $A \in \mathbf{R}^{+} S O(n)$. Finally, (3.8) with $q=l$ yields

$$
\left(\left|\nabla u^{j}\right|^{l}-|A|^{l}\right)^{+} \rightarrow 0 \text { in } L_{w}^{1}
$$

For $A=0$ this last inequality and homogeneity give (4.2). For $A \neq 0$, one can argue as in Step 3 of the proof of Theorem 1.1 or directly exploit the convexity of the map $A \rightarrow|A|^{l}$ to deduce $\left\|\nabla u^{j}-A\right\|_{L_{w}^{l}} \rightarrow 0$. The proof is complete.

The following example will show that the coercivity in Theorem 4.1 cannot be improved from $L_{w}^{n / 2}(\Omega)$ to $L^{n / 2}(\Omega)$. 
Example 4.2. Let $B_{r}$ be the open ball in $\mathbf{R}^{n}$ with radius $r>0$ and $A$ be an anticonformal matrix with $\operatorname{det} A=-1$. For any $\sigma \geq 1$, define

$$
u^{\sigma}(x)= \begin{cases}\left(\frac{1}{|x|}\right)^{1+\frac{1}{\sigma}} A x & 0<|x|<1 \\ (2-|x|) A x & 1 \leq|x|<2 .\end{cases}
$$

One easily sees that $u_{\sigma}(x)=0$ on $|x|=2$ and

$$
\nabla u^{\sigma}(x)= \begin{cases}A\left(\frac{1}{|x|}\right)^{1+\frac{1}{\sigma}}\left(I-\frac{\sigma+1}{\sigma} \frac{x}{|x|} \otimes \frac{x}{|x|}\right), & 0<|x|<1 \\ A\left((2-|x|) I-|x| \frac{x}{|x|} \otimes \frac{x}{|x|}\right), & 1 \leq|x|<2 .\end{cases}
$$

By virtue of this, an easy calculation shows that $u^{\sigma}$ belongs to $W_{0}^{1, n / 2}\left(B_{2} ; \mathbf{R}^{n}\right)$ and $\left\{\nabla u^{\sigma}\right\}$ is uniformly bounded in $L_{w}^{n / 2}\left(B_{2}\right)$. Note that

$$
\int_{B_{2}}\left(W\left(\nabla u^{\sigma}(x)\right)+d^{n / 2}\left(\nabla u^{\sigma}(x)\right)\right) d x \leq C<\infty, \quad \text { for all } 1 \leq \sigma \leq 2,
$$

however,

$$
\lim _{\sigma \rightarrow 1^{+}}\left\|\nabla u^{\sigma}\right\|_{L^{n / 2}\left(B_{1}\right)}=\infty \text {. }
$$

This shows the weak- $L^{n / 2}$ pseudo-norm cannot be replaced by $L^{n / 2}$ norm in the previous theorem.

\section{References}

[1] Acerbi, E. and Fusco, N. Semicontinuity problems in the calculus of variations, Arch. Rational Mech. Anal., 86, 125-145, (1984).

[2] Ball, J.M. Convexity conditions and existence theorems in nonlinear elasticity, Arch. Rational Mech. Anal., 63, 337403, (1977).

[3] Ball, J.M. Sets of gradients with no rank-one connections, J. Math. Pures Appl., 69, 241-259, (1990).

[4] Bojarski, B.V. and Iwaniec, T. Another approach to the Liouville's theorem, Math. Nachr, 107, 253-262, (1982).

[5] Ball, J.M. and James, R.D. Proposed experimental tests of a theory of fine microstructures and the two well problem, Phil. Trans. Roy. Soc. London, A, 338, 389-450, (1992).

[6] Ball, J.M. and Murat, F. $W^{1, p}$-Quasiconvexity and variational problems for multiple integrals, J. Funct. Anal., 58, 225-253, (1984).

[7] Chipot, M. and Kinderlehrer, D. Equilibrium configurations of crystals, Arch. Rational Mech. Anal, 103, 237-277, (1988).

[8] Dacorogna, B. Direct Methods in the Calculus of Variations, Springer-Verlag, Berlin, 1989.

[9] DiPerna, R.J. Compensated compactness and general systems of conservation laws, Trans. Am. Math. Soc., 292(2), 383-420, (1985).

[10] Donaldson, S.K. and Sullivan, D.P. Quasiconformal 4-manifolds, Acta Math., 163, 181-252, (1989).

[11] Evans, L.C. Quasiconvexity and partial regularity in the calculus of variations, Arch. Rational Mech. Anal., 95, 227-252, (1986).

[12] Fonseca, I. Variational methods for elastic crystals, Arch. Rational Mech. Anal., 97, 189-220, (1987).

[13] Gilbarg, D. and Trudinger, N.S. Elliptic Partial Differential Equations of Second Order, 2nd ed., Springer-Verlag, Berlin, 1984.

[14] Iwaniec, T. p-Harmonic tensors and quasiregular mappings, Ann. Math., 136, 589-624, (1992).

[15] Iwaniec, T. and Lutoborski, A. Integral estimates for null Lagrangians, Arch. Rational Mech. Anal., 125, 25-79, (1993).

[16] Iwaniec, T. and Martin, G. Quasiregular mappings in even dimensions, Acta Math., 170, 29-81, (1993).

[17] Iwaniec, T. and Sbordone, C. Weak minima of variational integrals, J. Reine Angew. Math., 454, 143-161, (1994).

[18] Iwaniec, T. and Šverák, V. On mappings with integrable dilatation, Proc. Am. Math. Soc., 118, 181-188, (1993). 
[19] Kinderlehrer, D. Remarks about equilibrium configurations of crystals, in Material Instabilities in Continuum Mechanics, Ball, J.M., Ed., Oxford University Press, 1988.

[20] Kinderlehrer, D. and Pedregal, P. Gradient Young measures generated by sequences in Sobolev spaces, J. Geom. Anal., 4(1), 59-90, (1994).

[21] Kohn, R.V. The relaxation of a double-well energy, Continuum Mech. Thermodyn., 3, 193-236, (1991).

[22] Morrey, C.B. Multiple Integrals in the Calculus of Variations, Springer-Verlag, Berlin, 1966.

[23] Müller, S. Higher integrability of determinants and weak convergence in $L^{1}$, J. Reine Angew. Math., 412, 20-34, (1990).

[24] Müller, S. On quasiconvex functions which are homogeneous of degree 1, Indiana Univ. Math. J., 41(1), 295-301, (1992).

[25] Müller, S. and Šverák, V. Attainment results for the two-well problem by convex integration, Geometric Analysis and the Calculus of Variations, (J. Jost, Ed.), Internat. Press, Cambridge, MA, 239-251, (1996).

[26] Reshetnyak, Yu.G. Stability Theorems in Geometry and Analysis, Kluwer Academic Publishers, Dordrecht, 1994.

[27] Stein, E. Singular Integrals and Differentiability Properties of Functions, Princeton University Press, Princeton, NJ, 1970.

[28] Šverák, V. Rank-one convexity does not imply quasiconvexity, Proc. R. Soc. Edin., 120(A), 185-189, (1992).

[29] Šverák, V. On regularity for the Monge-Ampère equation without convexity assumptions, preprint, 1992.

[30] Šverák, V. On Tartar's conjecture, Ann. Inst. H. Poincaré, Analyse Non Linéaire, 10(4), 405-412, (1993).

[31] Šverák, V. On the problem of two wells, in Microstructure and Phase Transition, Kinderlehrer, D., et al., Eds., Springer-Verlag, Berlin, 183-190, 1993.

[32] Šverák, V. Lower semicontinuity for variational integral functionals and compensated compactness, Proc. I.C.M., Zürich, (1994).

[33] Tartar, L. The compensated compactness method applied to systems of conservation laws, in Systems of Nonlinear Partial Differential Equations, Ball, J.M., Ed., NATO ASI Series, Vol. CIII, D. Reidel, 1983.

[34] Yan, B. Remarks about $W^{1, p}$-stability of the conformal set in higher dimensions, Ann. Inst. H. Poincaré, Analyse Non Linéaire, 13(6), 691-705, (1996).

[35] Yan, B. On rank-one convex and polyconvex conformal energy functions with slow growth, Proc. R. Soc. Edin., 127A, 651-663, (1997).

[36] Yan, B. On quasiconvex hulls of sets of matrices and strong convergence of certain minimizing sequences, preprint, 1993.

[37] Yan, B. and Zhou, Z. Stability of weakly almost conformal mappings, Proc. Amer. Math. Soc., 126, 481-489, (1998).

[38] Zhang, K. Biting theorems for Jacobians and their applications, Ann. Inst. H. Poincaré, Analyse Non Linéaire, 7, 345-365, (1990).

[39] Zhang, K. A construction of quasiconvex functions with linear growth at infinity, Ann. Scuola Norm. Sup. Pisa, 19(3), $313-326,(1992)$.

Received May 30, 1996

Max-Planck-Institute for Mathematics in the Sciences, Inselstr. 22-26, 04103 Leipzig, Germany e-mail: sm@mis.mpg.de

School of Mathematics, University of Minnesota, Minneapolis, MN

e-mail: sverak@math.umn.edu

Department of Mathematics, Michigan State University, East Lansing, MI e-mail: yan@math.msu.edu 